\title{
The Role of Futility Analyses in Alzheimer's Disease Clinical Trials
}

\author{
R. Doody \\ F. Hoffmann-La Roche/Genentech Product Development, Neuroscience, Basel, Switzerland and Genentech, Inc., South San Francisco, CA, USA \\ Corresponding author: Rachelle Doody, Grenzacherstrasse 124, 4070 Basel, Switzerland, Phone: +41 616870946 , Fax: +41 61 691 93 91, rachelle.doody@roche.com
}

$\mathbf{F}$ utility analyses in clinical trials are designed for all the right reasons: we want to stop exposing subjects to unnecessary risk and inconvenience if there is no chance that the intervention will provide them with benefit, and we want to direct resources toward more promising treatments.

However, there is no infallible formula or procedure that generates the needed analysis plan to accomplish these goals. Various methods have been proposed to calculate the probability of a statistically significant final result conditional upon interim data (Bayesian and nonBayesian approaches to conditional probability); or to define group sequential stopping boundaries (1).

In general, these approaches require that you know in advance what the treatment difference between treated and untreated patients should be and/or that you set arbitrary false-negative and false-positive rates, acknowledging that you may be wrong no matter what you do. These challenges are especially evident in a field like Alzheimer's disease (AD), where there are new mechanisms of action, trial designs, and outcome measures, and where few approved therapies exist to guide answers to these questions. Estimations or guesses to these unanswered questions affect the accuracy as well as the statistical power of the analysis.

The AD field has experienced successful, as well as unsuccessful, futility analyses. We learned from a futility analysis of the SCarlet RoAD trial of low-dose gantenerumab for prodromal $\mathrm{AD}$ that the trial was futile, which led to stopping that study as well as the Marguerite RoAD trial of the same low dose in patients with mild AD (2). This enabled us to learn more about the molecule in an open-label extension study, and to subsequently launch a larger, longer, global Phase 3 program, maximizing drug exposure with a five-fold higher dose (3-5). More recently, a futility analysis of the Phase 3 CREAD study of crenezumab allowed us to stop two global trials in early $\mathrm{AD}$, but to continue the Alzheimer's Prevention Initiative trial for delaying the onset or prevention of autosomal dominant $\mathrm{AD}$ in people who are PSEN1 E280A mutation carriers $(6,7)$.
Every futility analysis, like every clinical trial, involves a ratio of potential benefit for the analysis to potential risk for conducting it, with the risk being the possibility of stopping a trial even when the treatment has some degree of benefit, and even when the degree of benefit is of the magnitude that the trial was originally designed to show. We must continue to evolve our futility analysis approaches, just as we continue to evaluate new molecules and trial designs.

Disclosures: R. Doody is a current employee and shareholder of F. HoffmannLa Roche Ltd, Basel, Switzerland and Genentech, Inc., South San Francisco, CA, USA.

Open Access: This article is distributed under the terms of the Creative Commons Attribution 4.0 International License (http:/ / creativecommons.org/ licenses/by/4.0/), which permits use, duplication, adaptation, distribution and reproduction in any medium or format, as long as you give appropriate credit to the original author(s) and the source, provide a link to the Creative Commons license and indicate if changes were made.

\section{References}

1. Snapinn S, Chen M-G, Jiang Q, Koutsoukos T. Assessment of futility in clinical trials. Pharm Stat 2006;5:273-281.

2. Voyle N, Abi-Saab D, Klein G, et al. The effect of low doses of gantenerumab on amyloid and tau biomarkers in cerebrospinal fluid (CSF) in the Marguerite RoAD study. Alzheimers Dement 2018;14(Suppl):P240. O1-09-02.

3. Klein G, Delmar P, Hofmann C, et al. 24-month amyloid PET results of the gantenerumab high-dose open label extension studies. Alzheimers Dement 2018;14(Suppl):P240-P241. O1-09-03.

4. Abi-Saab D, Andjelkovic M, Pross N, et al. Update on the safety and tolerability of gantenerumab in the ongoing open-label extension (OLE) of the Marguerite RoAD study in patients with prodromal Alzheimer's disease (AD) after approximately 2 years of study duration. Alzheimers Dement 2018;14(Suppl):P241. O1-09-04.

5. Pross N, Ristic S, Andjelkovic N, et al. GRADUATE I and II better by design or how to optimize study designs by integrating learnings from previous studies. Oral presentation at the 14th International Conference on Alzheimer's \& Parkinson's Diseases (AD / PD) 2019; March 26-31, 2019; Lisbon, Portugal.

6. Tariot PN, Lopera F, Langbaum JB, et al. The Alzheimer's Prevention Initiative Autosomal-Dominant Alzheimer's Disease Trial: a study of crenezumab versus placebo in preclinical PSEN1 E280A mutation carriers to evaluate efficacy and safety in the treatment of autosomal-dominant Alzheimer's disease, including a placebo-treated noncarrier cohort. Alzheimers Dement (NY) 2018;4:150-160.

7. Roche. Roche to discontinue Phase III CREAD 1 and 2 clinical studies of crenezumab in early Alzheimer's disease (AD) - other company programmes in AD continue. 30 January 2019. https: / / www.roche.com/media/releases / med-cor-2019-01-30.htm. Accessed 13 December 2019. 